\title{
Memory for words in prose and their locations on the page
}

\author{
EUGENE A. LOVELACE \\ University of Virginia, Charlottesville, Virginia \\ and \\ STEPHEN D. SOUTHALL \\ Lynchburg College, Lynchburg, Virginia
}

\begin{abstract}
Two experiments are reported that establish that the recall of the within-page spatial location and the content of words from a prose passage are not functionally independent, i.e., that each can serve to cue recall of the other. Depriving college students of the spatial-location cues on a page by having them read a passage in the continuous form from a scroll significantly lowered word recall, whereas providing them with cues that reinstated the within-page location of material at time of test significantly raised recall of words. Providing the content of answers to the questions at time of recall was found to increase memory for location. The data are consistent with a conception of memory as a constellation of features such that recall of one of these features serves a cuing function to facilitate recall of other features.
\end{abstract}

Much of the recent work in memory for verbal materials assumes that even the individual word is not a unitary entity in memory, but rather is composed of a set of features or attributes (e.g., Brown \& McNeill, 1966; Wickens, 1970). A particular episodic memory may be viewed as a constellation of attributes. In this vein, Underwood (1969) noted that "when a memory is conceptualized as consisting of an ensemble of attributes, memory for an event per se has no psychological meaning because a memory without attributes is incapable of being remembered (retrieved). There is no 'corpus' which can be recalled directly" (p. 559). Spatial location continues to be identified as one of the fundamental attributes of such constellations (e.g., McCormack, 1976; Underwood, 1983). It appears to be a feature that is "automatically" encoded (Hasher \& Zacks, 1979; Schulman, 1973); instructing people that their memory for location also will be tested does not substantially improve spatial memory (e.g., Mandler, Seegmiller, \& Day, 1977; von Wright, Gebhard, \& Karttunen, 1975; Zechmeister, McKillip, Pasko, \& Bespalec, 1975).

The present research grew out of our observations that students answering quiz questions that called for only a key word, or a few words, from their text often told us that they knew where the word(s) they sought

The present report is based on dissertation research carried out by the second author under the direction of the first author at the University of Virginia; the first author, however, assumes primary responsibility for the present description of that research. Requests for reprints should be addressed to Eugene A. Lovelace, Psychology Department, Gilmer Hall, University of Virginia, Charlottesville, Virginia 22901. were located on the page when they were unable to recall the word(s). Several studies have shown that, when students read a prose passage, they are later able to assign material to the portion of the page on which it occurred, for example, eighths or quarters of the page, at a level that is consistently above chance, albeit modest in absolute level of accuracy (e.g., Christie \& Just, 1976; Rothkopf, 1971; Zechmeister \& McKillip, 1972). Zechmeister and McKillip also noted that students frequently report at the time of a test that they can recall (even "see") where a certain piece of information was located on the page, although they may not be able to recall the content. This knowledge of spatial location on the page is "incidental location," in the sense that one rarely is required to know location; tests of memory for prose typically deal only with content, not location. Such retrieval of some attributes, but not the ones required by the present memory task, presumably underlies the interesting phenomenon of "tip-of-thetongue" states (Brown \& McNeill, 1966) and "feelings of knowing" (Hart, 1965, 1967).

Knowing where something was on the page when the words that appeared there cannot be recalled, coupled with the more frequent ability to recall the content without knowing where it was located on the page, suggests that memory for content and for location are, in one sense, independent attributes of memory; that is, they are separable-one can occur without the other. There is also reason to believe, however, that when spatial location is encoded in such a way as to be linked to other attributes for which memory will be tested (usually semantic content), the spatial locations can be effective as a mnemonic device (e.g., Bower, 1970; 
Rawles, 1978; Yates, 1966). Rawles reminded us that, although psychologists have only recently begun to study "page location," it has long been widely employed as a memory aid by thespians, and was commented on by Charles Darwin in reply to Sir Francis Galton's queries concerning visualizing.

In several studies, the ability to accurately specify location has been shown to be correlated with the accuracy of recall of the semantic content of a sentence (e.g., Rothkopf, 1971; Zechmeister \& McKillip, 1972; Zechmeister et al., 1975). These authors have noted the following possible interpretations of this correlational evidence: (1) It might result from fluctuations of attention, such that, for those portions of the passage to which the individual attended carefully, he or she is able to recall both what was read and where it was located on the page, but, for other parts of the passage, he or she was "tuned out" and therefore reading became mechanical, and neither can be recalled;(2) the recall of the answer to one of the questions may, by providing more material, enhance the cue value over that of the question stem alone, and thus facilitate access to the spatial attribute; and (3) the recall of the spatial attribute may provide more of the constellation of features, and thus permit improved performance in answering content questions. Clearly these accounts are not mutually exclusive, and all three might contribute to the observed correlation.

The attentional account, although intuitively appealing and "simple," is neither interesting from a memorytheoretic point of view nor consistent with the observation by Zechmeister et al. (1975) that the recall of content was not differentially related to quadrant of the page, but that success at specifying location was. The other two explanations of the correlation, that memory for either content or spatial location can facilitate recall of the other, are both implied by any model of memory that assumes (1) that the coded memory representation for an event is a constellation of attributes, and (2) that the greater the number of attributes retrieved, the greater the cuing value for accessing additional attributes. Within this framework, the question of interest is whether certain attributes, in this case certain content words in a sentence and spatial location on the page, are functionally independent in their activation at the time of attempted recall or interact such that each may serve to facilitate activation of, or access to, the other.

Zechmeister and his associates have presented data that appear to support the notion that the codes for content and spatial attributes do not serve such a facilitative cuing function. Zechmeister and McKillip (1972, Experiment 2) found that when spatial knowledge was provided to the subjects, content recall was not affected. In their study, spatial information was provided at the time of recall by telling individuals the corner of the page in which it occurred; of course, this procedure may not have been adequate to reinstate reliably the location information. In a later study, they reported that when subjects "were provided the correct fill-in answers before attempting spatial recall," the accuracy of "spatial retention was not reliably changed" (Zechmeister et al., 1975, p. 51.).

The present studies, however, provide evidence that knowledge of location and memory for content are not functionally independent. Recall of content, as used here, refers not to the semantic gist of a sentence, but to the essentially verbatim recall of the specific key content word(s) of the queried sentence from a prose passage. Recall of within-page location required that individuals indicate on which quarter of the page the queried sentence appeared (we intend "within-page location cues" to include all the sources of information provided by any aspects of the spatial structure of the page). The approach taken in this research was two-fold: (1) to determine whether elimination, or marked reduction, of within-page location cues from the memory trace would result in a reduced level of recall of the content of the passage, and (2) to determine whether reinstatement of either precise location information or the content of the answer to the question at the time of recall would enhance the levels of content recall and of location recall, respectively.

In the current research, prior to the two major experiments aimed at these questions, two preliminary studies were conducted; they are summarized here, but are not described in detail. These studies replicated the major results of Rothkopf (1971) and Zechmeister and McKillip (1972) by finding reliable, but not high, levels of knowledge of spatial location, and a correlation of the accuracy of spatial location with correct recall. In the first of these two studies, using a passage and questions from Rothkopf's study and requesting location within quarters of the page, we found the probability of correct recall of spatial location, $P(L)$, to be .32 and the probability of correct recall of answers, $P(R)$, to the 32 questions to be .42 . The following conditional probabilities indicate that memory for the two types of information was related: The probability of correct location given a correct answer to the question, $P(L \mid R)$, was .43 , whereas the probability of correct location given an incorrect answer, $P(L \mid \bar{R})$, was .24 , essentially chance. Similarly, the probability of a correct answer to the question, given that location was correctly specified, $\mathrm{P}(\mathrm{R} \mid \mathrm{L})$, was .56 , but when location was incorrect, $P(R / \bar{L})$, this was only .35. Wilcoxon's matched-pairs signed-ranks tests applied to proportions for individuals showed that the accuracy of location recall was reliably greater when the answer was correct than when it was not, and that the accuracy of the answers was reliably greater when the location was correctly specified than when it was not. (All statistical tests referred to as significant have ps $<.05$.)

The second background study employed a 12-page typewritten passage containing factual information about the state of Alaska, drawn from an encyclopedia, 
and a 32-item short-answer completion test that was constructed by taking sentences from the passage and replacing critical facts with blanks. It is important to note two characteristics of these materials. First, this passage was constructed by selecting paragraphs such that the passage was essentially the concatenation of a great many discrete facts about the climate, geography, fiora, fauna, demography, and history of Alaska. There is no compelling logical order for presentation of this information: The paragraphs, and sometimes even the sentences within paragraphs, can be permuted without any apparent loss or change of meaning. Whereas Rothkopf (1971) also was interested in knowledge of location in the sense of early or late in the whole passage, we were concerned solely with within-page location and chose materials that minimized the extent to which content was tied to location due to the passage's structure, within the larger passage or within paragraphs. Analyses of the macrostructure of the passage (e.g., Deese, 1983; Kintsch, 1974) would indicate that the propositions tend to show coordinate, rather than strong super-/ subordinate, relations, and thus the position of a given sentence within the entire passage, and even within paragraphs, is of little value in cuing semantic content.

Second, the to-be-remembered item was typically a single key word, and the retrieval cue took the form of providing the whole sentence again, verbatim, with the to-be-remembered word(s) left blank for the individual to recall. The four forms of the passage differed with respect to the quarter of the first page in which the prose material began; thus, a given portion of the text (and therefore the same test sentence) occurred equally of ten, over forms, in each of the four quarters of the page.

The two conditions of this second background study differed with respect to reading instructions: One group was told to read the passage once carefully and not to go back over the materials, whereas the other was told to study the passage as they normally would for a test. The latter group took $39 \mathrm{~min}$, on average, to finish their reading, whereas the former took $27 \mathrm{~min}$, on average. Although memory for both location and content was slightly greater for those reading as they would for a test, the pattern of the results closely paralleled that for the traditional instructions to read through once carefully, and only the data from the latter group are described. The $P(R)$ was .52 , and the $P(L)$ was .33. The pattern of conditional probabilities was very similar to that seen in the study described above. The $P(L / R)$ was .37 , whereas the $\mathrm{P}(\mathrm{L} \mid \overline{\mathrm{R}})$ was .29 , and the $\mathrm{P}(\mathrm{R} \mid \mathrm{L})$ was .58 , whereas the $P(R / \bar{L})$ was .49 . Again, these differences were significant by Wilcoxon tests. Thus, in both background studies, the conditional probabilities indicated that recall of location was related to recall of content. However, the findings in these two preliminary studies simply provided additional evidence of a correlational sort, and did not differentiate between the three theoretical accounts presented above.
The rationale for the two major experiments reported here was to provide a more direct experimental test of the assumption that the recall of location information might function to facilitate content recall, and vice versa. In Experiment 1, it was hypothesized that depriving an individual of within-page location cues during reading of a prose passage would have detrimental effects on the level of content recall, since location information could not then be recalled at the time of test and serve a cue function to facilitate word recall. This was accomplished by having individuals read from a continuous scroll, which they advanced at their own rates, so that the passage had no pages as such. In Experiment 2, it was hypothesized that providing the location attribute at the time of test would facilitate word recall, and vice versa. Materials were presented in 12-page booklets. To test whether facilitation of word recall would occur if the spatial-location information could be activated at the time of test, performance on the same sentence fill-in questions when those sentences appeared at an arbitrary location on the page in a test booklet was compared with performance when they were presented at exactly the same location on the page that they had occupied for the study presentation. To assess whether providing the individual with the content answers to each of the fill-in questions would similarly enhance the recall of spatial location over the recall that could be achieved on the basis of the information contained in the question, performance on specifying the location on the page on the basis of the query sentence was compared with and without the words of the content answers filled in.

\section{EXPERIMENT 1}

\section{Method}

Subjects. Sixty students enrolled in introductory psychology classes at the University of Virginia participated for extra course credit.

Design. Twenty students served in each of three conditions, which differed in the spatial-location cues present when the passage was being studied. To minimize location cues in one condition, the passage was typed on a long, continuous sheet with no pages marked off. To permit the use of location cues for a second group, the passage was typed on pages that were joined end to end on the continuous paper scroll; in this continuous-with-pages procedure, the extra spacing at the top and bottom of each page was preserved, as was the page number, and a heavy black line was drawn where pages came together. To provide a baseline to determine whether reading from pages that were presented on a continuous scroll might itself change some aspect of performance, a control condition read the text passage in the traditional booklet form. Testing procedures were identical in all three conditions, except that individuals who read a continuous passage without pages could not, of course, be tested for within-page location information.

Materials. The 12-page, double-spaced typewritten passage on the state of Alaska employed in the second background study was used. For the continuous-without-pages condition (hereafter simply referred to as the continuous condition), the passage was typed on an $8 \frac{1}{2}$-in.-wide continuous sheet; for the continuous-with-pages and the booklet conditions, it was typed 
on $8^{1 / 2} \times 11$ in. pages, 28 lines per page. In the continuous-withpages condition, these pages were taped end to end on a continuous sheet such as that employed in the continuous condition. For the two conditions in which the passage was placed on a continuous scroll, the window allowed a little more than a page to be viewed at one time. The three versions of the passage were the same, line by line, and differed only in pagination procedures.

A 32-item short-answer-completion test was composed; from two to four questions were drawn from each page of the passage, with the total number of questions taken from each quarter of the pages being equal. Each question was a sentence taken verbatim from the passage, but with one or two words left blank for the individual to fill in. Pilot data from six individuals had indicated that the mean proportion of correct responses by individuals who had not read the passage would be near zero; that is, the questions called for answers that were not likely to be part of the subject's prior world knowledge.

Procedure. The students were randomly assigned to conditions and were run individually. Reading was self-paced; instructions indicated that a sentence could be reread if it had not been understood, but that whole pages or more were not to be reread once finished. They were told that they would be tested with short-answer fill-in questions requiring one or two words. Only after reading had been completed were those people in the continuous-with-pages and booklet conditions informed that they would be asked for the location of the material. Testing was also self-paced; test booklets contained four questions per page. Beside each question was a miniature diagram of a page with the quarters marked off and numbered; in the continuouswith-pages and booklet conditions, the individuals circled one of the numbers to report the location of the material on the page; individuals in the continuous condition were instructed to ignore those diagrams.

\section{Results and Discussion}

The method that the subjects used to advance the paper was observed to be very similar in the two continuous conditions; individuals in both groups advanced about a page of material and read that before advancing again. Although the total number of times the scroll was advanced was not recorded, we feel confident from our observations that it was very similar in these two conditions. There were no significant differences in mean reading times for the continuous-with-pages conditions $(17.5 \mathrm{~min})$, the continuous condition $(17.0 \mathrm{~min})$, or the booklet condition $(15.6 \mathrm{~min})$. The booklet and continuous-with-pages conditions did not differ appreciably in the number of items recalled, the means of individuals' proportions correctly recalled being .27 and .29 , respectively $[\mathrm{t}(38)=1.51, \mathrm{p}>.10]$, or in correct locations, for which the proportions were .30 and .29 $(t<1)$. In addition, the conditional probabilities for these two conditions never differed by more than $2 \%$ : The mean proportions for the booklet and continuous-with-pages conditions, respectively, were $\mathrm{P}(\mathrm{L} \mid \mathrm{R})$ $=.38$ and $.36, P(L \mid \bar{R})=.27$ and $.26, P(R \mid L)=.34$ and .36 , and $P(R \mid \bar{L})=.24$ and .26. From the similarity of values for the two conditions, it appears that there was no notable change in the processing of the prose passage as pages affixed to a continuous sheet or scroll, which the individual advanced by turning knobs, relative to the standard booklet format. Returning to the issue of whether the recall of each attribute was related to the recall of the other, the differences in $P(R \mid L)$ and $P(R \mid \bar{L})$ were significant in both the booklet and continuous- with-pages conditions by Wilcoxon tests, as were the differences in $P(L / R)$ and $P(L / \bar{R})$.

As predicted, the recall of content was found to be poorer in the continuous condition (.22) than in the continuous-with-pages condition (.29); this effect, roughly a $25 \%$ reduction in recall, was significant [ $t(38)$ $=2.14]$ and accounts for about $11 \%$ of the variance (eta squared $=.108$ ). It appears that, by minimizing the location information available in the continuous condition, the content recall was reduced. Further support for this interpretation is provided by the similarity of the proportion recalled in the continuous condition (.22) to that seen in the continuous-with-pages condition on those questions for which the individual could not provide the location $[\mathrm{P}(\mathrm{R} / \overline{\mathrm{L}})=.24]$; when location information was present during study but was not recallable by the individual at the time of test, the recall of word content was essentially the same as when no location information was present to be stored.

\section{EXPERIMENT 2}

This study tested two hypotheses: (1) reinstatement of position cues at the time of test should serve to enhance recall of the content of a prose passage, and (2) reinstatement of the content material should serve to improve the accuracy of location judgments. With respect to the latter hypothesis, Zechmeister et al. (1975) indicated in their general discussion that they had failed to find support for this hypothesis, but they did not provide procedural details of that research. Although Zechmeister and McKillip (1972, Experiment 2) failed to support the first hypothesis, their procedure for providing the location information was simply to prepare test booklets "with the correct corner of the page indicated for each question" (p. 450). The present study provided a more potent manipulation by reinstating the exact location on the page for the entire sentence from which the missing words were to be filled in. The current data provide support for both hypotheses: The experimental presentation of either attribute at the time of recall was found to enhance the recall of the other.

\section{Method}

Subjects. Sixty students enrolled in introductory psychology classes at the University of Virginia received extra credit for participation.

Design. Three groups of 20 students studied a passage under the study conditions described for the booklet condition in Experiment 1; in fact, one of these groups was the group in that condition, since those individuals served in a control condition that constituted a baseline for both experiments. The three groups, although treated identically during study, differed with respect to their testing procedures. Test conditions for the booklet condition were described in Experiment 1. For a second group, the answers to the questions were already filled in at the time of the test, and the individuals had to recall only location (the location condition); for the third group, the actual location on the page was shown to the individuals, who attempted to recall only the content (content condition). 
Materials. The same 12-page passage on Alaska was used. The test booklet for the location condition was the same as that for the booklet condition, except that for the former the content had already been typed on the blank line. For the content condition, a photocopy of the appropriate page of the passage was made for each question, and all of the words except those in the sentence that constituted the test question, were then individually blacked out with a felt marking pen; the critical word(s) were then covered to create the blank spaces to be filled in, and a second photocopy was made. This procedure should have maximized the reinstatement of all aspects of location on the page, including paragraph indentation and other format aspects. The test questions themselves were identical across conditions.

Procedure. All individuals were instructed as previously described for the booklet condition, except that on the test portion of the task, those in the location condition were told that they would be provided with the answers and that they were only to try to locate the sentence with respect to quarters of the page. In the content condition, the subjects were informed that the sentences were being shown in the exact locations where they had actually occurred and that they were to recall the content by filling in the blanks.

\section{Results and Discussion}

The total number of correct answers to questions in the content condition was greater than that for the booklet condition (means of 11.7 and 8.5 , respectively). This represents approximately a $38 \%$ increase in the number of correct answers, an effect that is significant $[t(38)=3.30]$, and accounts for about $22 \%$ of the variance (eta squared $=.223$ ). The mean proportion of correct content recall in the content condition was .36; this is very similar to $P(R / L)$ in the booklet condition, which was .34. This similarity is what would be expected if the current procedure for reinstating location information had effects very much like those resulting from accurate self-cuing by successful location recall. Precise reinstatement of the configuration of within-page location cues apparently results in greater recall of the content of a prose passage.

The total number of correct location judgments for the location condition was greater than that for the booklet condition (means of 11.4 and 9.6, respectively). This $19 \%$ increase was significant $[\mathrm{t}(38)=2.35]$ and accounts for about $13 \%$ of the variance (eta squared $=.127$ ). The mean proportion of correct location judgments in the location condition was .36 , which is very similar to $P(L \mid R)$ in the booklet condition (.38). The facilitation of correct location judgments resulting from knowledge of the content is about the same, regardless of whether that content was recalled by the individual or provided by the researcher.

\section{GENERAL DISCUSSION}

In terms of explicit task demands, any knowledge of within-page location of the material is "incidental" to performance of the task in most memory tests for prose material. Data in previous studies have shown that there is a relationship between memory for location and content, but this correlation could be accounted for by variation in attention while reading, and no previous experimental data have indicated a causal link. The present studies made it clear, however, that the recall of within-page spatial information and of the content words from a prose passage are not functionally independent. That is, manipulation of the availability of location or content information at the time of test was shown to have a significant effect on recall of the other attribute.

In Experiment 1, the continuous-passage procedure precluded the presence of within-page spatial-location cues; this significantly lowered the level of content recall. In Experiment 2, the reinstatement of exact within-page spatial location at the time of the test was found to significantly facilitate content recall. These two findings made it clear that knowledge of spatial location can serve to cue the recall of the content of a prose passage. Experiment 2 also demonstrated that providing the individual with the content answers to questions can facilitate location recall. Under the present conditions, location cues appeared to have somewhat greater cue value to aid content recall than did the provision of content answers aid the recall of location; however, the two types of recall differ in measurement scale (e.g., chance or guessing levels), and thus direct comparison is not justified.

In Experiment 2, the procedure for reinstating the within-page location of the sentence also provided, on some occasions, information about where the sentence fell in a paragraph as well as its distance from top and bottom edge of the page. This was intentional in that this research intended to assess the benefits of reinstating all aspects of within-page location that might be available if the individual were able to recreate an effective image of the page at the time of test. The extent to which within-page location cues, in the present global sense, include effects due to knowledge of position of the statement in the paragraph structure remains to be explored. The effects of spatial-location information obtained in Experiment 1, however, cannot be explained by text structure or paragraph location effects. Furthermore, in one respect, the present data give little evidence of such effects of paragraph structure in Experiment 2. If knowledge of the location of a sentence within a paragraph facilitated correct content recall, then such recall would be greater for questions from the latter portion of the page, since the probability that the preceding paragraph break appears on that page increases the further down the page a sentence appears. No such systematic relationship between quarter of the page and correct content recall was found. Of course, it must be noted that the present passage was selected so as not to possess strong hierarchical structure.

For some mnemonists, images employing spatial location can be extremely powerful mnemonics (e.g., Luria's, 1968, S) and there is evidence that many individuals are capable of training themselves to possess exact knowledge of location on the page for extended amount of prose material, as evidenced by Stratton's (1917) description of the Shass Pollak, Polish Jews who 
could specify any word in the Talmud from a specification of the page number and the word's position on the page. The present data confirm, as several researchers have suggested, that the storage of location appears to occur "automatically" to some extent for all of us. As Schulman (1983) noted, words on a page may be particularly difficult things to encode spatially, relative to naturally occurring objects in a visually rich environment. Furthermore, the general spatial frames in which these words are encoded are not distinctive; the page contours of successive pages are very similar. Thus, the present use of imagery for words on a page, although modest in the absolute level of its effect, is the more impressive because the conditions are not those that should promote the most effective use of imagery. Recent research by Shebilske and Rotondo (1981) demonstrated that the enhancement of spatial cues, resulting from a special format and typography, improved recall of material from a biology textbook; when systematically queried about how they felt the special typography affected their recall, many individuals gave answers that indicated that visual imagery had played an important role. The increasing use of computer technology in arranging the format and typography of prose permits greater flexibility and should lead to an improved use of spatial characteristics to enhance comprehension and retention. Furthermore, the widespread use of video displays in the production and editing of prose in current word-processing devices makes one aspect of these data particularly interesting. Whereas, in many situations involving memory for prose, knowledge of location on the page may be considered an "incidental" attribute, many editing operations on a word processor are facilitated by knowledge of location, which permits rapidly finding specific material to be modified.

In summary, the present results are consistent with a general memory model in which the within-page spatial location of words is treated as one of several attributes that the reader of a prose passage stores in memory and in which the recall of a given attribute is facilitated by the recall of other attributes that are components of some constellation of attributes constituting the memory for that prose passage.

\section{REFERENCES}

Bowen, G. H. Analysis of a mnemonic device. American Scientist, 1970, 58, 496-510.
Brown, R., \& McNeill, D. The "tip of the tongue" phenomenon. Journal of Verbal Learning and Verbal Behavior, 1966, 5, 325-337.

Christie, J., \& Just, M. A. Remembering the location and content of sentences in a prose passage. Journal of Educational Psychology, 1976, 68, 702-710.

DEESE, J. E. Thought into speech: The psychology of a language. Englewood Cliffs, N.J: Prentice-Hall, 1983.

HaRT, J. T. Memory and the feeling of knowing experience. Journal of Educational Psychology, 1965, 56, 208-216.

HaRT, J. T. Memory and the memory-monitoring process. Journal of Verbal Learning and Verbal Behavior, 1967, 6, 685-691.

Hasher, L., \& Zacks, R. T. Automatic and effortful processes in memory. Journal of Experimental Psychology: General, 1979, 108, 356-388.

KINTSCH, W. The representation of meaning in memory. New York: Wiley, 1974.

LuRiA, A. R. The mind of a mnemonist. New York: Basic Books, 1968.

Mandler, J. M., Seegmiller, D., \& Day, J. On the coding of spatial information. Memory \& Cognition, 1977, 5, 10-16.

McCormack, P. D. Language as an attribute of memory. Canadian Journal of Psychology, 1976, 30, 238-248.

RAwles, R. E. The past and present of mnemotechny. In M. M. Gruneberg, P. E. Morris, \& R. N. Sykes (Eds.), Practical aspects of memory. London: Academic Press, 1978.

RothкоPF, E. Z. Incidental memory for location of information in text. Journal of Verbal Learning and Verbal Behavior, 1971, 10, 608-613.

Schulman, A. I. Recognition memory and the recall of spatial location. Memory \& Cognition, 1973, 1, 256-260.

Schulman, A. I. Maps and memorability. In D. R. Rogers \& J. Sloboda (Eds.), The acquisition of symbolic skills. New York: Plenum Press, 1983.

ShEBILsKe, W. L., \& Rotondo, J. A. Typographical and spatial cues that facilitate learning from textbooks. Visible Language, $1981,15,41-54$.

Stratton, G. M. The mnemonic feat of the "Shass Pollak." Psychological Review, 1917, 24, 244247.

Undenwood, B. J. Attributes of memory. Psychological Review, $1969,76,559-573$.

UNDE rwOOD, B. J. Attributes of memory. Glenview, Ill: Scott, Foresman, 1983.

von Wright, J. M., Gebhard, P., \& Karttunen, M. A developmental study of the recall of spatial location. Journal of Experimental Child Psychology, 1975, 20, 181-190.

Wickens, D. D. Encoding categories of words: An empirical approach to meaning. Psychological Review, 1970, 77, 1-15.

YATES, F. A. The art of memory. Chicago: University of Chicago Press, 1966.

Zechmeiste R, E. B., \& McKILLIP, J. Recall of place on the page. Journal of Educational Psychology, 1972, 63, 446-453.

Zechmeisten, E. B., McKillip, J., Pasko, S., \& Bespalec, D. Visual memory for place on the page. Journal of General Psychology, 1975, 92, 43-52.

(Manuscript received June 17, 1983; revision accepted for publication June 22, 1983.) 